\title{
Tuberculosis of the Sternoclavicular Joint: Report of 3 New Cases Observed in Abidjan
}

\author{
Mohamed Diomandé*, Mariam Gbané-Koné, Baly Ouattara, Tamo Estelle Mégné, \\ Edmond Eti, Marcel N'zué Kouakou \\ Department of Rheumatology, University Hospital Center of Cocody, Abidjan, Côte d'Ivoire \\ Email: diomandemohamed48@yahoo.fr, mariamgbane05@yahoo.fr, baly ouattara@yahoo.fr, \\ megnees@yahoo.fr, etiedmondoutlook@yahoo.fr, mnkouakou@yahoo.fr
}

Received 20 December 2014; accepted 15 January 2015; published 20 January 2015

Copyright (C) 2015 by authors and Scientific Research Publishing Inc.

This work is licensed under the Creative Commons Attribution International License (CC BY).

http://creativecommons.org/licenses/by/4.0/

cc) (7)

Open Access

\begin{abstract}
The sternoclavicular joint is a rare site of osteoarticular tuberculosis. We now report three new cases of sternoclavicular tuberculosis. The report concentrates mostly on immunocompromised patients, notably two with HIV infection and one case marked by its acute onset which is rather original because, in this case, there was a swollen sternoclavicular joint with normal $\mathrm{X}$-ray and it was the CT-scan which showed the lesions. The etiological diagnosis was made in the first case by polymerase chain reaction and in the second case by identification of Mycobacterium tuberculosis. In the third case, the diagnosis was presumptive and the outcome on anti-tuberculous drugs was satisfactory. Sternoclavicular joint tuberculosis is unusual; the painful swelling of that joint should lead to realize a performed imaging such as CT-scan.
\end{abstract}

\section{Keywords}

Osteoarticular Tuberculosis, Sternoclavicular Joint, CT-Scan, Abidjan

\section{Introduction}

The tuberculosis is all around the world. The initial contamination is by airways. Osteoarticular tuberculosis (OAT) results from hematogenous spread of Mycobacterium tuberculosis (MT) from a primitive visceral home, most often lung. There are two situations: the spread of MT is old and the occurrence of TOA corresponds to a local reactivation or the spread of MT is recent and there is an active and remote localization. The advent of HIV

${ }^{*}$ Corresponding author.

How to cite this paper: Diomandé, M., Gbané-Koné, M., Ouattara, B., Mégné, T.E., Eti, E. and N'zué Kouakou, M. (2015) Tuberculosis of the Sternoclavicular Joint: Report of 3 New Cases Observed in Abidjan. Open Journal of Rheumatology and Autoimmune Diseases, 5, 1-5. http://dx.doi.org/10.4236/ojra.2015.51001 
infection saw appearance of unusual ostearticular tuberculosis. The sternoclavicular joint tuberculosis (SCJT) is very rare: $1 \%$ of peripheral OAT [1]. Few cases of sternoclavicular joint arthritis were described in the literature and concerned mostly septic arthritis [2]. We report three new cases of SCJT observed in Rheumatology Department of University Hospital Center of Cocody in Abidjan, emphasizing on clinical features and the importance of the performed imaging. One of the cases was marked by its acute onset which was rather original.

\section{Observation 1}

A 52-year-old patient with no particular past medical history has presented spontaneous inflammatory neck pain which started one month ago, associated with paresthesia of the upper limbs. After one week, appeared a swollen right sternoclavicular joint leading to functional impotence of the right upper limb. Other system review was normal. Physical examination revealed multidirectional stiffness of the cervical spine and hypereflexia of the four limbs without motor or sensitivity impairment. Sternoclavicular joint was swollen, tender, cold and the rest of exam was normal. On biology, the erythrocyte sedimentation rate (ESR) was $62 \mathrm{~mm}$ in the first hour, C reactive protein (CRP) $125.4 \mathrm{mg} / \mathrm{l}$ and the PCR realized on the abscess taken from the swollen joint, was positive and revealed Mycobacterium tuberculosis; HIV serology was negative. The chest X-ray was normal. The CTscan of the neck revealed bifocal spondylodiscitis at the level of C4-C5 and C6-C7 with epidural inflammation and CT-scan of the right sternoclavicular joint revealed were osteitis and arthritis (Figure 1). The diagnosis of the SCJT and Pott's disease was made. The evolution was good under antitubercular treatment which associated rifampicin (R), isoniazid (H), ethambutol (E), pyrazinamid (Z); two months with RHZE followed by ten months of RH.

\section{Observation 2}

A 53-year-old man was hospitalized for a swollen painful right sternoclavicular joint for three months. There was no improvement after three weeks of antibiotics but rather an increased volume of the joint associated with ipsilateral functional impotence of the upper limb. The tuberculosis infection signs were present (night fever, night sweating, altered general status); other systemic inquiry was normal. On physical examination, there was a swollen painful sternoclavicular joint (Figure 2) with liquid liking from skin fistula. The analysis of the liquid showed acid fast bacillae. The ESR was $95 \mathrm{~mm}$, the CRP was $12.5 \mathrm{mg} / \mathrm{l}$, Intra-dermal reaction to the tuberculin (IDRt) was $15 \mathrm{~mm}$ and HIV serology was positive. The chest X-ray was within normal limits. The thoracic CT-scan revealed an osteitis and arthritis sternoclavicular right joint with soft tissue abscess. He received twelve months antitubercular drugs according to the protocol 2 months RHZE/10 months RH and the outcome were favorable.

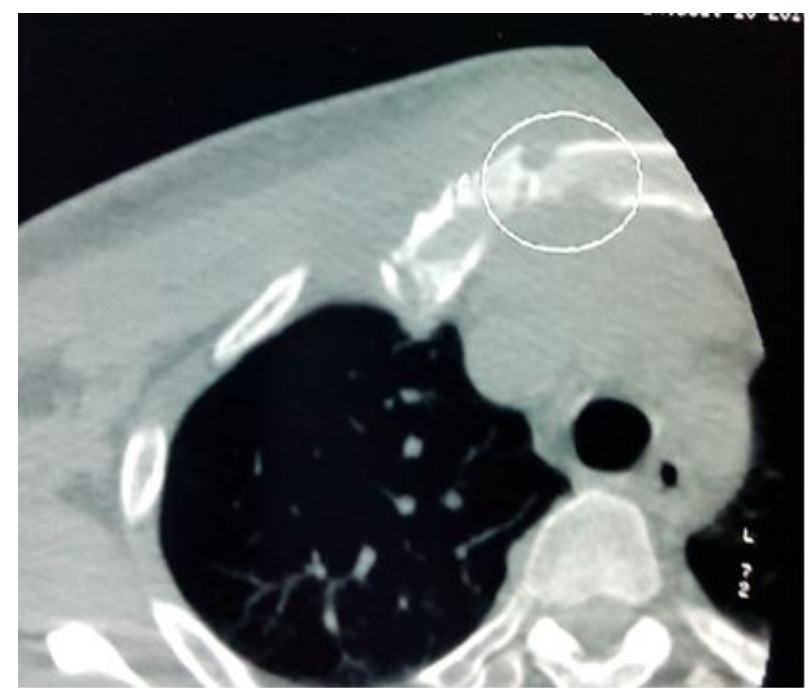

Figure 1. CT-scan of the right sternoclavicular joint showing an osteitis and arthritis (patient 1). 


\section{Observation 3}

A 51-year-old man, with a past medical history of treated pulmonary tuberculosis three years ago was admitted in rheumatology, because of swollen of right sternoclavicular joint, lasting twelve months with functional impotence of the homolateral upper limb. He took unknown drugs by his own initiative. These symptoms were associated with night fever, gradual weight lost, night sweating. The sternoclavicular joint was swollen, tender and painful. There was no particularity on visceral examination. The CRP was $24 \mathrm{mg} / \mathrm{l}$, the ESR was $60 \mathrm{~mm}$, the IDRt was measured at $18 \mathrm{~mm}$ and the HIV serology was positive. The chest X-ray didn't permit clearly to identify the sternoclavicular joint, the CT-scan showed an osteitis and arthritis with abscess of soft tissues (Figure 3). The diagnosis of sternoclavicular joint tuberculosis was retained and the evolution was satisfactory after twelve months of treatment with antitubercular drugs according to the protocol of two months with RHZE followed by ten months of RH.

\section{Discussion}

The osteoarticular tuberculosis represents $2 \%$ to $5 \%$ of the overall tuberculosis [3]. The sternoclavicular localization is extremely rare (1\% of all peripheral joint tuberculosis) [1]. This scarcity was noticed on studies

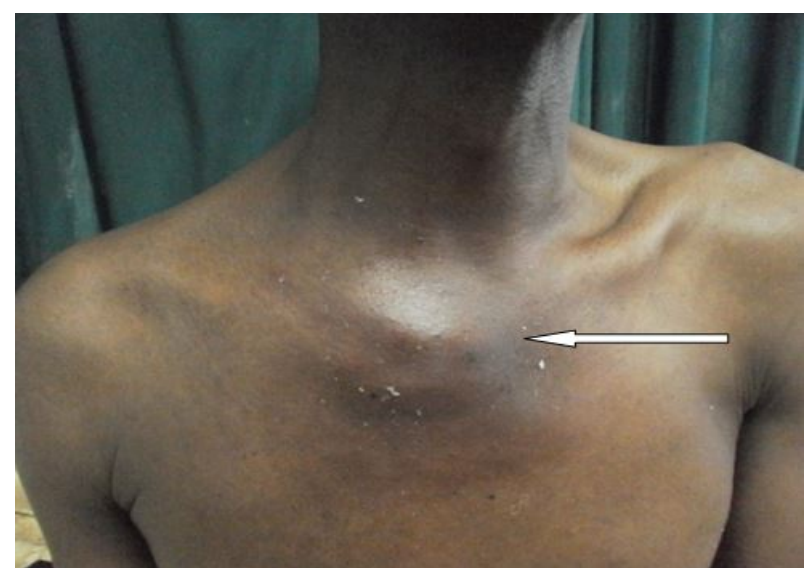

Figure 2. Fluctuating swelling of the right sternoclavicular joint which has fistulized later (patient 2).

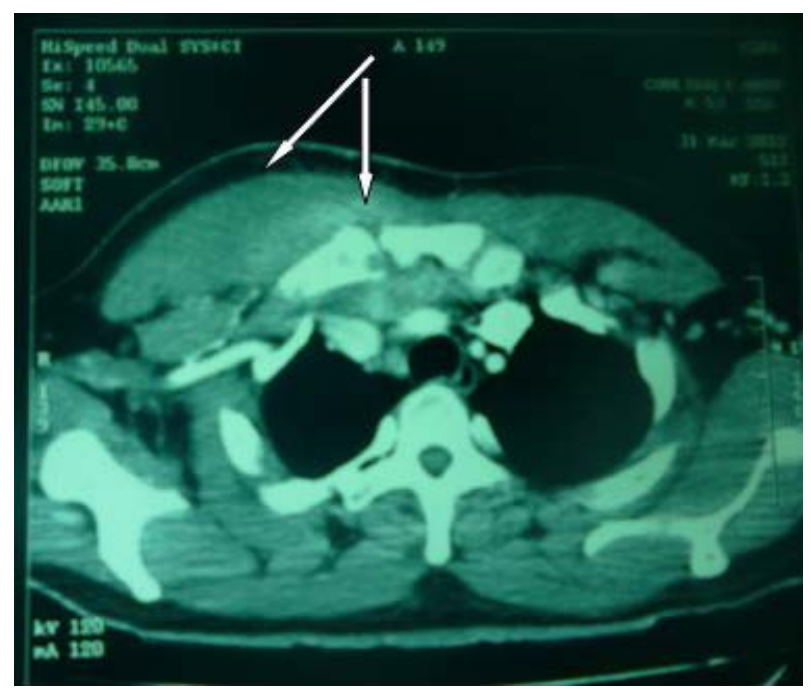

Figure 3. Thoracic CT-scan showing an osteitis and arthritis of the right sternoclavicular joint with soft tissue abscess surrounding (patient 3). 
carried out by Tuli [4] who identified only 7 cases of SCJT out of 1070 OAT. In the literature few cases of sternoclavicular arthritis are due to community germs, particularly Staphylococcus aureus compared to Mycobacterium tuberculosis. The diagnosis is sometimes delayed as seen in our three cases (mean duration of 4.5 months) because of slow onset of the few symptoms of disease [1].

These unusual presentations of OAT appear in immunocompromised patients notably those with HIV as observed in two of our patients. Other causes of immune deficiency such as diabetes, cortisteroid therapy, sickle cell anemia, cancer, cytotoxic drugs, auto-immune diseases, chronic renal failure, can facilitate the onset of SCJT, but those risk factors were not seen in our patients. Usually SCJT is monoarticular with painful localized swollen joint as seen in our cases and it is associated in $20 \%$ to $40 \%$ to either pulmonary or lymph nodes involvement [3]. In three cases there was no visceral involvement. Only the third patient had a past medical history of pulmonary tuberculosis, usually found in one third of the cases [5].

Concerning imaging, such localization is difficult to be seen on the X-ray because of the overlapping structures, thus making the CT-scan and (magnetic resonance imaging) MRI indispensible. The CT-scan can show lytic lesions, joint destruction, arthritis and soft tissue abscess. The MRI will show early soft tissue and bone involvement. In our cases the CT-scan revealed lesions that were not seen on X-rays. The CT-scan and MRI are reference imaging [6] as revealed in our cases. The diagnosis was confirmed by identification of Mycobacterium tuberculosis in the first case directly in the pus and in the second case, by the positivity of the PCR in the pus. The PCR is a recent technique with a specificity ranging from $92 \%$ to $98 \%$ allowing early diagnosis, seems new and unknown in the Sub-Saharan Africa [3]. In the third case the epidemiology, clinical, paraclinical arguments and obviously the evolution helped in diagnosis according to the Eti, et al.'s research results [7]. The first case is characterized by its acute onset (3 weeks), usually it is sub acute or chronic [8]-[10]. This acute onset will make to think first of all of sternoclavicular arthritis due to community germs. Other differential diagnosis could be rheumatoid arthritis, spondylarthropathies, articular chondrocalcinosis (pseudogout) or Tietze's syndrome [6].

Concerning treatment, the antitubercular drugs based on the association rifampicin (R), isoniazid (H), ethambutol (E) and pyrazinamide (Z); according to the protocol of two months of RHZE followed by ten months of $\mathrm{RH}$, remains widely used in our environment and is sufficient for the healing.

\section{Conclusion}

The sternoclavicular joint tuberculosis is exceptional. It can be revealed by unusual acute onset. The swollen sternoclavicular joint should draw attention and require investigations where CT scan and/or MRI are particularly important.

\section{Conflict of Interest}

None.

\section{References}

[1] Kurtz, B., Hauss, P.A., Druesne, L. and Chassagne, P. (2005) Tuberculosis Arthritis of the Sternoclavicular Joint. Revue de Medecine Interne, 26, 249-245.

[2] Enarson, D.J., Fujii, M., Nakielna, E.M. and Grzybowsky, S. (1979) Bone and Joint Tuberculosis: A Continuing Problem. Canadian Medical Association Journal, 120, 139-145.

[3] Pertuiset, E. (2004) Peripheral Bone and Joint Tuberculosis. Encyclopedie Medicochirurgical-Rhumatologie Orthopédie, 1, 463-486. http://dx.doi.org/10.1016/j.emcrho.2004.08.003

[4] Tuli, S.M. (1993) Tuberculosis of the Skeletal System (Bone, Joints, Spine and Bursal Sheaths). Jaypee Brothers Medical Publisher, New Delhi.

[5] Daniel, T.M. and Debanne, S.M. (1987) The Serodiagnosis of Tuberculosis and Other Mycobacterial Diseases by Enzyme-Linked Immunosorbent Assay. American Review of Respiratory Disease, 135, 1137-1151.

[6] Ankit, S., Jyotirmoy, P., Partho, S.K. and Nirodh, B.D. (2010) Tuberculosis of Sternoclavicular Joint-Uncommon Manifestation of a Common Disease. Journal of Medicine, 11, 102-104.

[7] Eti, E., Daboiko, J.C., Brou, K.F., Ouali, B., Koffi, K.D. and Kouakou, N.M. (2010) Vertebral Tuberculosis: Our Experience from a Study of 147 Cases in the Rheumatology Department of the University Hospital of Cocody, Abidjan, Côte d'Ivoire. Medecine d'Afrique Noire, 57, 287-292. 
[8] Cibère, J. (2000) Rheumatology: Four. Acute Monoarthritis. Canadian Medical Association Journal, 162, 1577-1583.

[9] Watts, H.G. and Lifeso, R.M. (1996) Tuberculosis of Bones and Joints. The Journal of Bone Joint Surgery American Volume, 78, 288-298.

[10] Payne, K. and Yang, J. (2002) Osteoarticular Tuberculosis: A Case Report and Discussion. Canadian Medical Association Journal, 166, 628-630. 
Scientific Research Publishing (SCIRP) is one of the largest Open Access journal publishers. It is currently publishing more than 200 open access, online, peer-reviewed journals covering a wide range of academic disciplines. SCIRP serves the worldwide academic communities and contributes to the progress and application of science with its publication.

Other selected journals from SCIRP are listed as below. Submit your manuscript to us via either submit@scirp.org or Online Submission Portal.
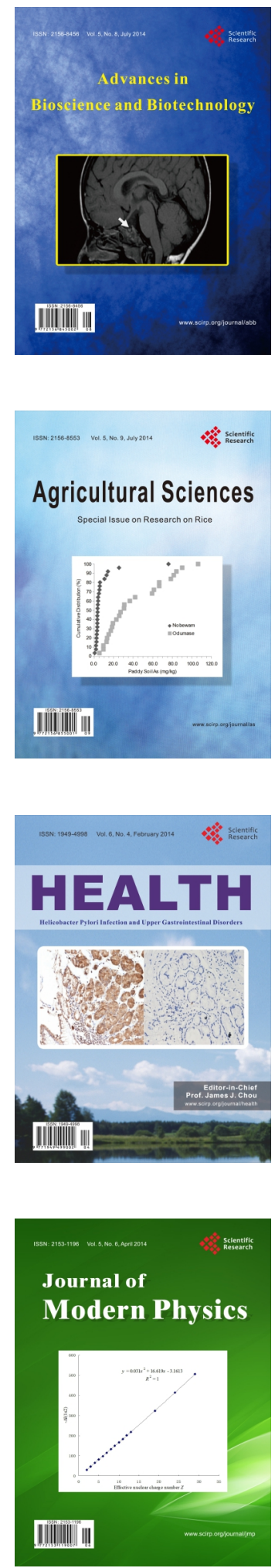
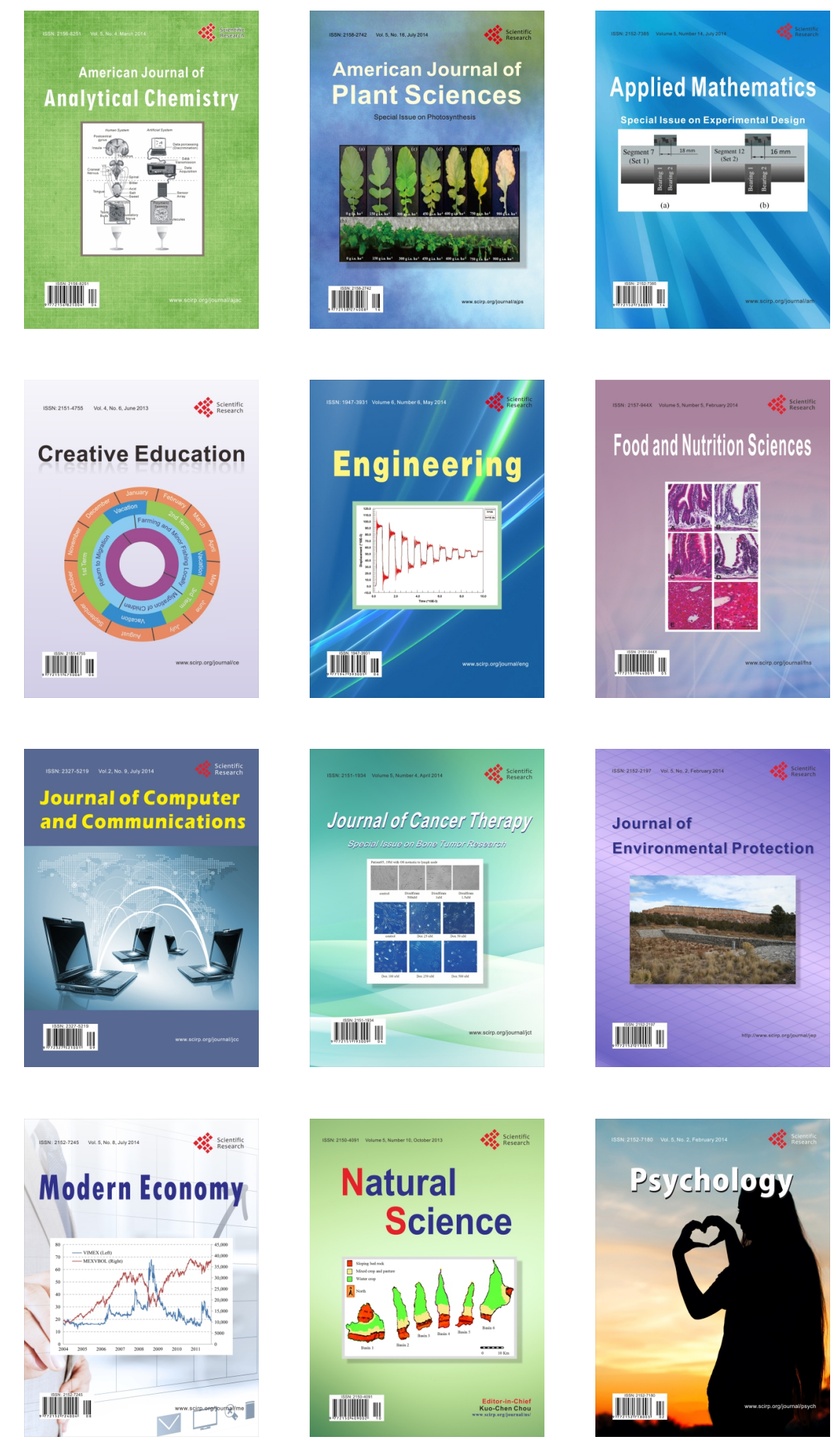